\title{
Observation of the Mass Difference between Neutral Charm-Meson Eigenstates
}

\author{
R. Aaij et al. \\ (LHCb Collaboration)
}

(Received 8 June 2021; accepted 8 July 2021; published 7 September 2021)

\begin{abstract}
A measurement of mixing and $C P$ violation in neutral charm mesons is performed using data reconstructed in proton-proton collisions collected by the LHCb experiment from 2016 to 2018, corresponding to an integrated luminosity of $5.4 \mathrm{fb}^{-1}$. A total of 30.6 million $D^{0} \rightarrow K_{\mathrm{S}}^{0} \pi^{+} \pi^{-}$decays are analyzed using a method optimized for the measurement of the mass difference between neutral charmmeson eigenstates. Allowing for $C P$ violation in mixing and in the interference between mixing and decay, the mass and decay-width differences are measured to be $x_{C P}=[3.97 \pm 0.46$ (stat) \pm 0.29 (syst) $] \times 10^{-3}$ and $y_{C P}=[4.59 \pm 1.20$ (stat) \pm 0.85 (syst) $] \times 10^{-3}$, respectively. The $C P$-violating parameters are measured as $\Delta x=[-0.27 \pm 0.18$ (stat) \pm 0.01 (syst) $] \times 10^{-3}$ and $\Delta y=[0.20 \pm 0.36$ (stat) \pm 0.13 (syst) $] \times 10^{-3}$. This is the first observation of a nonzero mass difference in the $D^{0}$ meson system, with a significance exceeding seven standard deviations. The data are consistent with $C P$ symmetry and improve existing constraints on the associated parameters.
\end{abstract}

DOI: 10.1103/PhysRevLett.127.111801

Neutral charm mesons propagating freely can change (oscillate) into their own antiparticles, as the mass eigenstates are linear combinations of the flavor eigenstates. These flavor-changing neutral currents do not occur at tree level in the standard model (SM) and allow for hypothetical particles of arbitrarily high mass to contribute significantly to the process. This can affect the mixing of mesons and antimesons such that measurements of these processes can probe physics beyond the SM [1-4].

The mass eigenstates of charm mesons can be written as $\left|D_{1,2}\right\rangle \equiv p\left|D^{0}\right\rangle \pm q\left|\bar{D}^{0}\right\rangle$, where $p$ and $q$ are complex parameters and, in the limit of charge-parity $(C P)$ symmetry, $\left|D_{1}\right\rangle\left(\left|D_{2}\right\rangle\right)$ is defined as the $C P$ even (odd) eigenstate. Mixing of flavor eigenstates is described by the dimensionless parameters $x \equiv\left(m_{1}-m_{2}\right) c^{2} / \Gamma$ and $y \equiv\left(\Gamma_{1}-\Gamma_{2}\right) /(2 \Gamma)$, where $m_{1(2)}$ and $\Gamma_{1(2)}$ are the mass and decay width of the $D_{1(2)}$ state, respectively, and $\Gamma$ is the average decay width [5]. In $D^{0}$ and $\bar{D}^{0}$ decays to a common final state, $f, C P$ violation in mixing manifests itself if $|q / p| \neq 1$ or in the interference between mixing and decay if $\phi_{f} \equiv \arg \left(q \bar{A}_{f} / p A_{f}\right) \neq 0$. Here $A_{f}\left(\bar{A}_{f}\right)$ denotes the amplitude of the decay process $D^{0} \rightarrow f\left(\bar{D}^{0} \rightarrow f\right)$. In the $D^{0} \rightarrow K_{\mathrm{S}}^{0} \pi^{+} \pi^{-}$decay studied in this Letter, $C P$ violation in the decay $\left(\left|A_{f}\right|^{2} \neq\left|\bar{A}_{f}\right|^{2}\right)$ is not considered,

\footnotetext{
*Authors are listed at the end of this Letter.

Published by the American Physical Society under the terms of the Creative Commons Attribution 4.0 International license. Further distribution of this work must maintain attribution to the author(s) and the published article's title, journal citation, and DOI. Funded by SCOAP ${ }^{3}$.
}

as in the SM it is negligible for the doubly Cabibbosuppressed (DCS) and Cabibbo-favored (CF) amplitudes contributing to this process. With this assumption, the $C P$ violating phase is independent of the final state, $\phi_{f} \approx \phi \approx$ $\arg (q / p)[6,7]$.

The current world average of the mixing and $C P$-violating parameters yields $x=(3.7 \pm 1.2) \times 10^{-3}, y=\left(6.8_{-0.7}^{+0.6}\right) \times 10^{-3}$, $|q / p|=0.951_{-0.042}^{+0.053}$, and $\phi=-0.092_{-0.079}^{+0.085}$ [8]. Measurements using decays such as $D^{0} \rightarrow K^{+} \pi^{-}$have resulted in precise measurements of $y$ and have allowed for the observation of mixing $[9,10]$. However, the data remain marginally compatible with $x=0$ and are consistent with $C P$ symmetry. Theoretical predictions for the mixing parameters are of similar magnitude but less precise [11,12], while predictions of the $C P$-violating phase are around 0.002 [13] and are well below the current experimental precision.

Sensitivity to the mixing and $C P$-violating parameters is offered by the self-conjugate, multibody $D^{0} \rightarrow K_{\mathrm{S}}^{0} \pi^{+} \pi^{-}$ decay [14-18]. Inclusion of the charge-conjugate process is implied unless stated otherwise. This final state is accessible in both $D^{0}$ and $\bar{D}^{0}$ decays and leads to interference between the mixing and decay amplitudes, as demonstrated pictorially in Fig. 1. The dynamics of the decay are expressed as a function of two invariant masses following

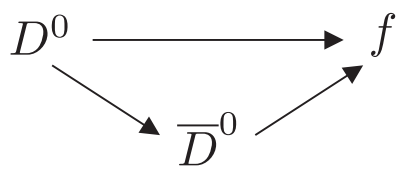

FIG. 1. Depiction of the interference of mixing and decay if a $D^{0}$ and a $\bar{D}^{0}$ meson decay to a common final state $f$. 
the Dalitz-plot formalism, in which a three-body decay is parameterized by a pair of two-body invariant masses $[19,20]$. The squared invariant mass $m^{2}\left(K_{\mathrm{S}}^{0} \pi^{ \pm}\right)$is denoted as $m_{ \pm}^{2}$ for $D^{0}$ decays and $m_{\mp}^{2}$ for $\bar{D}^{0}$ decays. A mixture of DCS and CF decay amplitudes results in large variations of the strong phase and, with mixing, causes a decay-time evolution of the density of decays across the phase space. A joint analysis of the Dalitz-plot and decay-time distributions may be used to determine the mixing parameters. Splitting the sample by flavor of the charm meson at production probes for $C P$-violating effects. Usage of multibody decay modes is typically challenging, as it requires knowledge of the variation of the hadronic parameters and excellent control of efficiencies, resolutions, and background effects.

This Letter reports on a measurement of the mixing and $C P$ violation parameters in $D^{0} \rightarrow K_{\mathrm{S}}^{0} \pi^{+} \pi^{-}$decays using the "bin-flip" method [21], a model-independent approach that obviates the need for detailed models of the efficiency, resolution, and contributing amplitudes. Mixing and $C P$ violation are parametrized by $z_{C P}$ and $\Delta z$, which are defined by $z_{C P} \pm \Delta z \equiv-(q / p)^{ \pm 1}(y+i x)$. The results are expressed in terms of the $C P$-even mixing parameters $x_{C P} \equiv-\operatorname{Im}\left(z_{C P}\right)$ and $y_{C P} \equiv-\operatorname{Re}\left(z_{C P}\right)$, and of the $C P$ violating differences $\Delta x \equiv-\operatorname{Im}(\Delta z)$ and $\Delta y \equiv-\operatorname{Re}(\Delta z)$. Conservation of $C P$ symmetry implies $x_{C P}=x, y_{C P}=y$, and $\Delta x=\Delta y=0$. The method has already been employed by the LHCb collaboration, yielding the single most precise measurement of $x_{C P}$ and $\Delta x$ [18].

In the bin-flip method, data are partitioned into disjoint regions (bins) of the Dalitz plot, which are defined to preserve nearly constant strong-phase differences $\Delta \delta\left(m_{-}^{2}, m_{+}^{2}\right)$ between the $D^{0}$ and $\bar{D}^{0}$ amplitudes within each bin [22]. Two sets of eight bins are formed symmetrically about the $m_{+}^{2}=m_{-}^{2}$ bisector, as illustrated in Fig. 2 . The region satisfying $m_{+}^{2}>m_{-}^{2}$, which includes regions dominated by the CF $D^{0} \rightarrow K^{*}(892)^{-} \pi^{+}$decay, is given a positive index $+b$, while the opposite region, where the relative contribution from decays following an oscillation is enhanced, is given a negative index $-b$. The data are further split into 13 bins of decay time, chosen such that the bins are approximately equally populated. The squared-mass and decay-time resolutions are typically $0.006 \mathrm{GeV}^{2} / c^{4}$ and $60 \mathrm{fs}$, respectively, which are smaller than the bin sizes used. Thus, they are neglected and accounted for in the systematic uncertainties.

For each decay-time interval $(j)$, the ratio of the number of decays in each negative Dalitz-plot bin $(-b)$ to its positive counterpart $(+b)$ is measured. The usage of ratios minimizes the need for precise modeling of the efficiency variation across phase space and decay time. For small mixing parameters and $C P$-conserving decay amplitudes, the expected ratios for initially produced $D^{0}\left(\bar{D}^{0}\right)$ mesons, $R_{b j}^{+}\left(R_{b j}^{-}\right)$, are [21]

$$
R_{b j}^{ \pm} \approx \frac{r_{b}+r_{b} \frac{\left\langle t^{2}\right\rangle_{j}}{4} \operatorname{Re}\left(z_{C P}^{2}-\Delta z^{2}\right)+\frac{\left\langle t^{2}\right\rangle_{j}}{4}\left|z_{C P} \pm \Delta z\right|^{2}+\sqrt{r_{b}}\langle t\rangle_{j} \operatorname{Re}\left[X_{b}^{*}\left(z_{C P} \pm \Delta z\right)\right]}{1+\frac{\left\langle t^{2}\right\rangle_{j}}{4} \operatorname{Re}\left(z_{C P}^{2}-\Delta z^{2}\right)+r_{b} \frac{\left\langle t^{2}\right\rangle_{j}}{4}\left|z_{C P} \pm \Delta z\right|^{2}+\sqrt{r_{b}}\langle t\rangle_{j} \operatorname{Re}\left[X_{b}\left(z_{C P} \pm \Delta z\right)\right]}
$$

The parameter $r_{b}$ is the value of $R_{b j}$ at $t=0$, while $X_{b}$ is the amplitude-weighted strong-phase difference between opposing bins. Finally, $\langle t\rangle_{j}\left(\left\langle t^{2}\right\rangle_{j}\right)$ corresponds to the average (squared) decay time in each positive Dalitz-plot

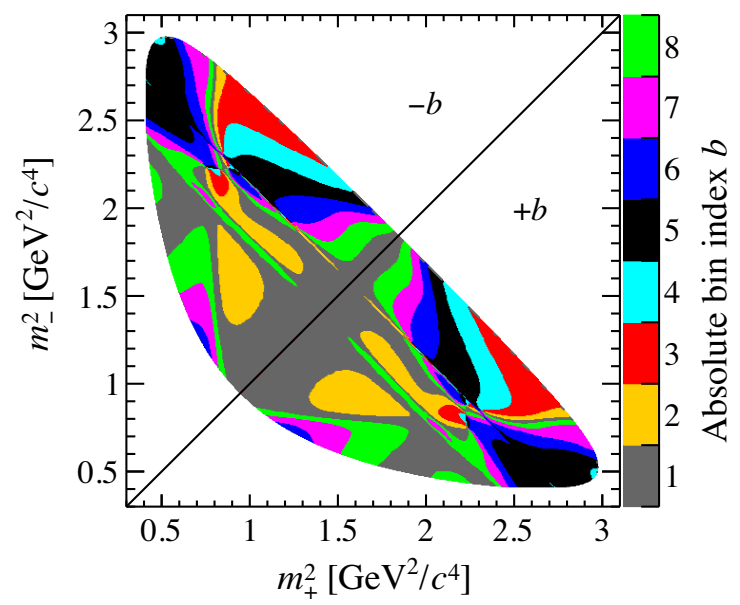

FIG. 2. "Binning" of the $D^{0} \rightarrow K_{\mathrm{S}}^{0} \pi^{+} \pi^{-}$Dalitz plot. Colors indicate the absolute value of the bin index $b$. region where the mixed contribution is negligible, in units of the $D^{0}$ lifetime $\tau=\hbar / \Gamma$ [5], calculated directly from background-subtracted data. The other parameters are determined from a simultaneous fit of the observed $R_{b j}^{ \pm}$ ratios, in which external information on $c_{b} \equiv \operatorname{Re}\left(X_{b}\right)$ and $s_{b} \equiv-\operatorname{Im}\left(X_{b}\right)[22,23]$ is used as a constraint.

Samples of $D^{0} \rightarrow K_{\mathrm{S}}^{0} \pi^{+} \pi^{-}$decays are reconstructed from proton-proton $(p p)$ collisions collected by the $\mathrm{LHCb}$ experiment from 2016 to 2018 , corresponding to an integrated luminosity of $5.4 \mathrm{fb}^{-1}$. The strong-interaction decay $D^{*+} \rightarrow D^{0} \pi^{+}$is used to identify the flavor of the neutral charm meson at production. Throughout this Letter, $D^{*+}$ indicates the $D^{*}(2010)^{+}$meson and soft pion indicates the pion from its decay. The LHCb detector $[24,25]$ is a single-arm forward spectrometer covering the pseudorapidity range $2<\eta<5$, designed for the study of particles containing $b$ or $c$ quarks.

Decays of $K_{S}^{0} \rightarrow \pi^{+} \pi^{-}$are reconstructed in two different categories, the first involving $K_{S}^{0}$ mesons that decay early enough for the pions to be reconstructed in all tracking detectors and the second containing $K_{S}^{0}$ mesons that decay 
later such that track segments of the pions cannot be formed in the vertex detector, which surrounds the $p p$ interaction (primary vertex) region, resulting in a worse momentum resolution. The latter category contains more candidates but has slightly worse mass and decay-time resolution as well as larger efficiency variations.

The online event selection consists of a hardware stage, selecting events based on calorimeter and muon detector information, followed by two software stages. In the first software stage, the pion pair from the $D^{0}$ decay is required to satisfy criteria on momenta and final-state chargedparticle displacements from any primary vertex for at least one pion (one-track) or both together with a vertex quality requirement (two-track). The second software stage fully reconstructs $D^{*+} \rightarrow D^{0} \pi^{+}, D^{0} \rightarrow K_{S}^{0} \pi^{+} \pi^{-}$candidates using further requirements on particle identification, momenta, and track and vertex quality. Specific ranges of displacement and invariant mass are imposed on the reconstructed $D^{0}$ and $K_{S}^{0}$ candidates. Because of differing efficiencies, the sample is split into four categories, depending on whether or not the $K_{S}^{0}$ meson is reconstructed in the VELO and whether or not they satisfy the one-track requirement.

Offline, a kinematic fit constrains the tracks to form vertices according to the decay topology, the $K_{S}^{0}$ candidate mass to the known value [5], and the $D^{*+}$ candidate to a primary vertex [26]. In the reconstruction of the Dalitz-plot coordinates, an additional constraint on the $D^{0}$ candidate mass to the known value improves the resolution. Charm mesons originating from the decays of $b$ hadrons are suppressed by requiring that the $D^{0}$ and soft pion candidates originate from a primary vertex. Candidates are rejected if two of the reconstructed tracks use the same hits in the vertex detector. About $6 \%$ of the candidates are from collision events in which multiple candidates are reconstructed, usually by pairing the same $D^{0}$ candidate with different soft pions. When this occurs, one candidate is chosen randomly, and the rest are removed from the sample.

Signal yields are determined by fitting the distribution of the mass difference between the $D^{*+}$ and $D^{0}$ candidates, denoted as $\Delta m$. The signal probability density function is empirically described by a combination of a Johnson $S_{U}$ distribution [27] and two Gaussian functions, one of which shares a mean with the Johnson $S_{U}$. The background is dominated by real $D^{0}$ decays incorrectly combined with a charged particle not associated with a $D^{*+}$ decay, and is modeled with a smooth phase space-like model, $\theta\left(\Delta m-m_{\pi}\right) e^{-c\left(\Delta m-m_{\pi}\right)}\left(\Delta m-m_{\pi}\right)^{\alpha}$, where $\theta(x)$ is the Heaviside step function, $m_{\pi}$ is the charged-pion mass [5], and $\alpha$ and $c$ are free parameters. Figure 3 shows the $\Delta m$ distribution of the entire sample, from which the fit identifies $(30.585 \pm 0.011) \times 10^{6}$ signal decays. This represents a factor of 15 larger yield compared to the previous measurement.

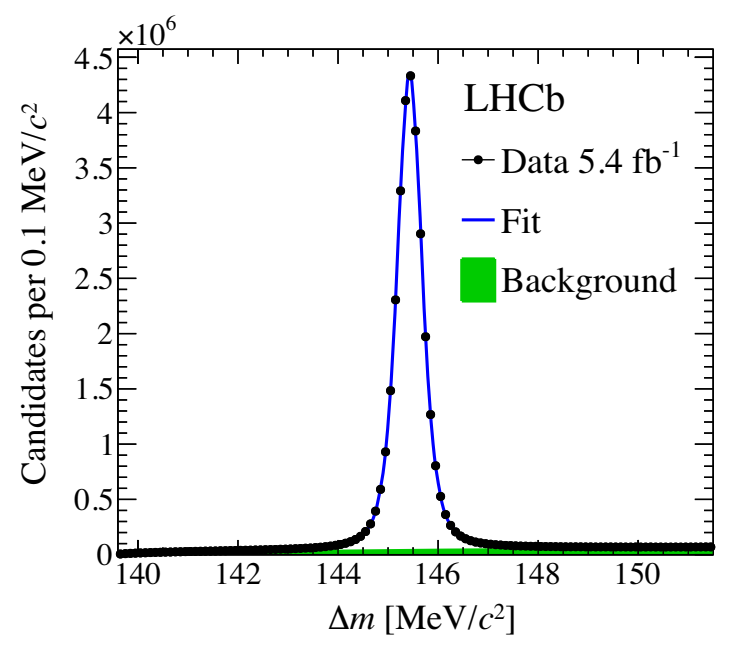

FIG. 3. Distribution of $\Delta m$ for the selected $D^{*+} \rightarrow$ $D^{0}\left(\rightarrow K_{S}^{0} \pi^{+} \pi^{-}\right) \pi^{+}$candidates. The projection of the fit result is superimposed.

To determine the yields used to form the ratios $R_{b j}^{ \pm}$, separate fits are performed for each set of Dalitz-plot and decay-time bins $b j$. The signal model assumes the same parameters for each pair of positive and negative Dalitz-plot bins and fixes some parameters from a fit integrated over decay time. Fits are performed independently for $D^{0}$ and $\bar{D}^{0}$ candidates, as well as for each of the four data subsamples. The measured signal yields are then corrected for two effects that do not cancel in the ratio: experimentally induced correlations between the phase space and decay time and charge-dependent efficiencies (detection asymmetries).

Online requirements on the displacement and momenta of the $D^{0}$ decay products introduce efficiency variations that are correlated between the phase-space coordinates and the $D^{0}$ decay time. The effect depends predominantly on the invariant mass of two pions from the $D^{0}$ decay, with the efficiency to reconstruct the candidates at low values decreasing significantly at low $D^{0}$ decay times. This can bias the measured yield ratios and produce mixing-like trends. To remove this bias, an approach that estimates the relative efficiencies using data is developed. The Dalitz plot is divided into small, rectangular-like regions formed symmetrically across the bisector. Note that these include the portions above and below the bisector, unlike the bins shown in Fig. 2. In the limit of $C P$ symmetry, the contribution of mixing to such symmetric regions depends only on $y_{C P}$ and the hadronic parameters of the $D^{0}$ decay [21]. As oscillations result in a migration of decays from one side of the Dalitz plot to the other, and the regions are symmetric with respect to the bisector, there is no effect from $x_{C P}$. Given a set of inputs for $y_{C P}$ and the hadronic parameters, the contribution of mixing to the decay-time distributions of these regions can be accounted for, such that the remaining differences between regions come from 
the efficiency correlations. Relative efficiency maps that align the decay-time distributions in all these symmetric regions can then be calculated. Per-candidate weights assigned by the efficiency maps are integrated over the data in each bin using the sPlot method [28] with $\Delta m$ as the discriminating variable. This provides correction factors for each of the fitted signal yields.

In calculating the efficiency maps, the strong phase variation within a Dalitz-plot bin is approximated as constant, such that it can be described by the external inputs $\left(s_{b}\right)$. As $y_{C P}$ and $s_{b}$ are parameters of the fit, the correction maps and corresponding correction factors are calculated for a range of values. The smallness of mixing results in smooth variations of the correction factors for a given Dalitz-plot bin, which allows for precise interpolation between the calculated points with polynomials. These polynomials are then incorporated into the fit as a correction that depends on $y_{C P}$ and $s_{b}$. The correction is calculated for each yield ratio but is averaged over the initial flavor of the candidates. The procedure has been validated with pseudoexperiments and a systematic uncertainty is assigned due to the approximation that $s_{b}$ is constant within a bin.

Corrections are also applied to take into account detection asymmetries. Because of utilizing ratios of yields, the analysis is insensitive to detection asymmetries of the $K_{S}^{0}$, as well as the soft pion used to tag the flavor of the candidate. However, the kinematics of the pions produced in the $D^{0}$ decay depend on the Dalitz-plot coordinate and $D^{0}$ flavor. This can result in asymmetric efficiency variations for $D^{0}$ and $\bar{D}^{0}$ candidates that imitate $C P$ violation. The two-track $\pi^{+} \pi^{-}$asymmetry, $A_{\text {det }}\left(\pi^{+} \pi^{-}\right)$, is determined by measuring detection asymmetries in control samples of $D_{s}^{+} \rightarrow \pi^{+} \pi^{+} \pi^{-}$and $D_{s}^{+} \rightarrow \phi \pi^{+}$decays, in which the $\phi$ meson is reconstructed through a $K^{+} K^{-}$pair. A randomly chosen $\pi^{+}$in the $D_{s}^{+} \rightarrow \pi^{+} \pi^{+} \pi^{-}$decay is paired with the $\pi^{-}$to form a proxy for the $\pi^{+} \pi^{-}$pair of interest. The $D_{s}^{+} \rightarrow$ $\phi \pi^{+}$sample is used to cancel asymmetries induced from the remaining $\pi^{+}, A_{\mathrm{det}}\left(\pi^{+}\right)$, and other sources, such as the trigger selection, $A_{\text {trigger }}\left(D_{s}^{+}\right)$, and the production of $D_{s}^{+}$ and $D_{s}^{-}$mesons in $p p$ collisions, $A_{\text {prod }}\left(D_{s}^{+}\right)$. For asymmetries of $\mathcal{O}(1 \%)$, the raw asymmetries $A_{\text {meas }}$ can be approximated as

$$
\begin{aligned}
A_{\text {meas }}\left(D_{s}^{+} \rightarrow \pi^{+} \pi^{+} \pi^{-}\right) \approx & A_{\text {det }}\left(\pi^{+} \pi^{-}\right)+A_{\text {det }}\left(\pi^{+}\right) \\
& +A_{\text {prod }}\left(D_{s}^{+}\right)+A_{\text {trigger }}\left(D_{s}^{+}\right), \\
A_{\text {meas }}\left(D_{s}^{+} \rightarrow \phi \pi^{+}\right) \approx & A_{\text {det }}\left(\pi^{+}\right)+A_{\text {prod }}\left(D_{s}^{+}\right) \\
& +A_{\text {trigger }}\left(D_{s}^{+}\right) .
\end{aligned}
$$

The difference of the two measured asymmetries gives the detection asymmetry of the $\pi^{+} \pi^{-}$pair. The control samples are weighted to match the kinematics of the pions from the $D^{0} \rightarrow K_{\mathrm{S}}^{0} \pi^{+} \pi^{-}$sample. This weighting is done separately for each Dalitz-plot bin. The detection asymmetries are of the order of $10^{-3}$ and are used as corrections to the measured yields. They are included as constraints in the fit along with the associated covariance matrix $\Delta V_{\text {asym }}$ describing uncertainties coming from the limited size of the calibration samples.

The mixing parameters are determined by minimizing a least-squares function

$$
\begin{aligned}
\chi^{2} \equiv & \sum_{+,-} \sum_{b, j} \frac{\left[N_{-b j}^{ \pm}-R_{+b j}^{ \pm} N_{+b j}^{ \pm} /\left(C_{b j}\left(1 \pm \Delta A_{b}\right)\right)\right]^{2}}{\left(\sigma_{-b j}^{ \pm}\right)^{2}+\left[R_{+b j}^{ \pm} \sigma_{+b j}^{ \pm} /\left(C_{b j}\left(1 \pm \Delta A_{b}\right)\right)\right]^{2}} \\
& +\sum_{b, b^{\prime}}\left(X_{b}^{\mathrm{EXT}}-X_{b}\right)\left(V_{\mathrm{EXT}}^{-1}\right)_{b b^{\prime}}\left(X_{b^{\prime}}^{\mathrm{EXT}}-X_{b^{\prime}}\right) \\
& +\sum_{b, b^{\prime}}\left(\Delta A_{b}^{\mathrm{asym}}-\Delta A_{b}\right)\left(\Delta V_{\mathrm{asym}}^{-1}\right)_{b b^{\prime}}\left(\Delta A_{b^{\prime}}^{\text {asym }}-\Delta A_{b^{\prime}}\right),
\end{aligned}
$$

where the yields $N$ and their measured uncertainties $\sigma$ are scaled by factors for the correlation removal, $C_{b j}$, and detection asymmetry correction, $\Delta A^{b} \equiv A_{\mathrm{det}}^{b}\left(\pi^{+} \pi^{-}\right)-$ $A_{\text {det }}^{-b}\left(\pi^{+} \pi^{-}\right)$. The different subsamples are fitted simultaneously, separated between $D^{0}$ and $\bar{D}^{0}$ flavors denoted as + and -, including all decay-time intervals $j$ and Dalitzplot bins $b$. The parameters $X_{b}$ are constrained with a Gaussian penalty term using the values $X_{b}^{\mathrm{EXT}}$ and covariance matrix $V_{\text {EXT }}$ from a combination of CLEO and BESIII measurements $[22,23]$. In the fit, the parameters $r_{b}$ are determined independently for each subsample, as they are affected by the sample-specific variation of the efficiency over the Dalitz plot [21]. To avoid experimenter's bias, the values of $x_{C P}, y_{C P}, \Delta x$, and $\Delta y$ were not examined until the full procedure had been finalized. Figure 4 shows the yield ratios with fit projections overlaid for each of the eight Dalitz-plot bins. Deviations from constant values are due to mixing. The fit projection when $x_{C P}$ is fixed to zero is also included and shows the inability of a nonzero $y_{C P}$ value to produce the deviations on its own. Also shown are the differences of ratios between $D^{0}$ and $\bar{D}^{0}$ decays, where a significant slope would indicate $C P$ violation.

Systematic uncertainties are assessed from ensembles of pseudoexperiments. These use the $D^{0} \rightarrow K_{\mathrm{S}}^{0} \pi^{+} \pi^{-}$model of Ref. [29] to describe the amplitude at $t=0$, and the decaytime dependence is incorporated for a range of values of the mixing and $C P$ violation parameters. Different sources of systematic uncertainty are included and the effect on the measured parameters evaluated [30]. The dominant systematic uncertainty on the mixing parameters comes from reconstruction and selection effects and amounts to $0.20 \times$ $10^{-3}\left(0.76 \times 10^{-3}\right)$ for $x_{C P}\left(y_{C P}\right)$. This includes neglecting the decay-time and $m_{ \pm}^{2}$ resolutions and efficiencies, as well as the correction to remove the efficiency correlations. The most important effect for $y_{C P}$ is the approximation of the strong phase to be constant within each bin in the procedure to remove correlations. Contamination from $b$-hadron 

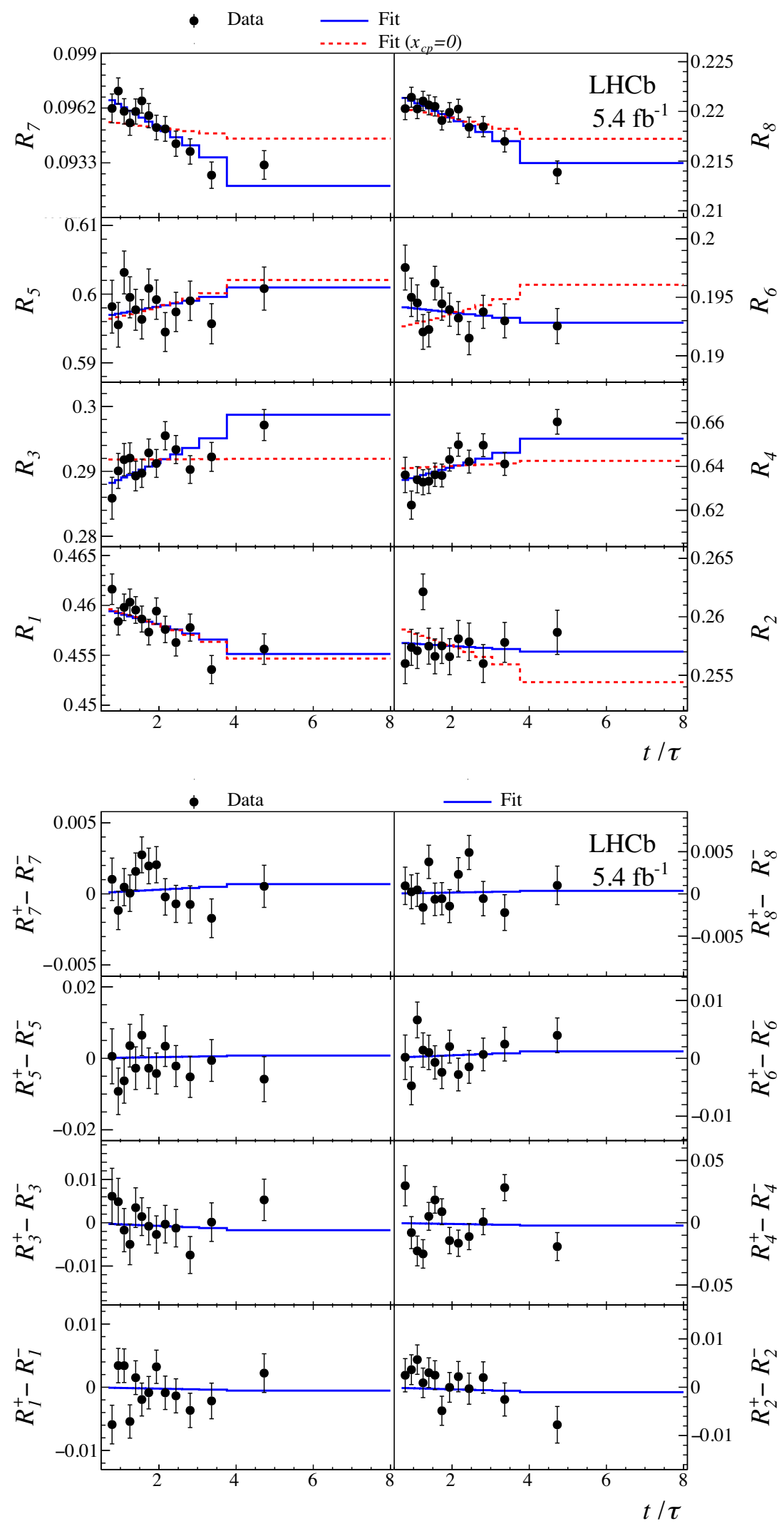

FIG. 4. (Top) $C P$-averaged yield ratios and (bottom) differences of $D^{0}$ and $\bar{D}^{0}$ yield ratios as a function of $t / \tau$, shown for each Dalitzplot bin with fit projections overlaid. 
decays contributes $0.20 \times 10^{-3}\left(0.15 \times 10^{-3}\right)$ to the $x_{C P}$ $\left(y_{C P}\right)$ uncertainty. Potential mismodeling in the signal yield fits contributes $0.36 \times 10^{-3}$ to the $y_{C P}$ uncertainty. Timedependent detection asymmetries are present mainly in bins that give the best sensitivity to $\Delta y$, resulting in a systematic uncertainty of $0.12 \times 10^{-3}$.

The consistency of the results is tested by repeating the analysis in subsets of the data, divided according to magnet polarity, trigger and $K_{S}^{0}$ category, data-taking period, $D^{*+}$ meson kinematics, and other categories. The largest variation occurs for the value of $x_{C P}$ as a function of $D^{*+}$ meson pseudorapidity, where the compatibility, considering statistical uncertainties only, amounts to a $p$-value of $1.5 \%$, depending on the details of the sample split, whereas the overall $p$-value for all $x_{C P}$ observed variations is above $8 \%$. The observed variations of the observables $x_{C P}, y_{C P}, \Delta x$, and $\Delta y$ are all consistent with statistical fluctuations.

The mixing and $C P$ violation parameters are measured to be

$$
\begin{aligned}
x_{C P} & =(3.97 \pm 0.46 \pm 0.29) \times 10^{-3}, \\
y_{C P} & =(4.59 \pm 1.20 \pm 0.85) \times 10^{-3}, \\
\Delta x & =(-0.27 \pm 0.18 \pm 0.01) \times 10^{-3}, \\
\Delta y & =(0.20 \pm 0.36 \pm 0.13) \times 10^{-3},
\end{aligned}
$$

where the first uncertainty is statistical and the second systematic [30]. The statistical uncertainty contains a subleading component due to the limited precision of the external measurements of the strong phases and control samples used for the detection asymmetry. This amounts to approximately $(0.23,0.66,0.04$, and 0.08$) \times 10^{-3}$ for $x_{C P}$, $y_{C P}, \Delta x$, and $\Delta y$, respectively. The measurements are statistically limited, though the systematic uncertainty on $y_{C P}$ is comparable to the statistical uncertainty. The results are used to form a likelihood function of $x, y,|q / p|$, and $\phi$ using a likelihood-ratio ordering that assumes the observed correlations to be independent of the true parameter values $[30,31]$. The best fit point is

$$
\begin{aligned}
x & =\left(3.98_{-0.54}^{+0.56}\right) \times 10^{-3}, \\
y & =\left(4.6_{-1.4}^{+1.5}\right) \times 10^{-3}, \\
|q / p| & =0.996 \pm 0.052, \\
\phi & =0.056_{-0.051}^{+0.047} .
\end{aligned}
$$

In summary, a measurement of mixing and $C P$ violation in $D^{0} \rightarrow K_{\mathrm{S}}^{0} \pi^{+} \pi^{-}$decays has been performed with the binflip method, using $p p$ collision data collected by the $\mathrm{LHCb}$ experiment and corresponding to an integrated luminosity of $5.4 \mathrm{fb}^{-1}$. This resulted in the first observation of a nonzero value of the mass difference $x$ of neutral charm meson mass eigenstates with a significance of more than seven standard deviations, and significantly improves limits on mixing-induced $C P$ violation in the charm sector.

We express our gratitude to our colleagues in the CERN accelerator departments for the excellent performance of the LHC. We thank the technical and administrative staff at the LHCb institutes. We acknowledge support from CERN and from the national agencies: CAPES, CNPq, FAPERJ, and FINEP (Brazil); MOST and NSFC (China); CNRS/ IN2P3 (France); BMBF, DFG, and MPG (Germany); INFN (Italy); NWO (Netherlands); MNiSW and NCN (Poland); Institul de Fizica Atomica (MEN/IFA) Romania; MSHE (Russia); MICINN (Spain); SNSF and SER (Switzerland); NASU (Ukraine); STFC (United Kingdom); and DOE NP and NSF (USA). We acknowledge the computing resources that are provided by CERN, IN2P3 (France), KIT and DESY (Germany), INFN (Italy), SURF (Netherlands), PIC (Spain), GridPP (United Kingdom), RRCKI and Yandex LLC (Russia), CSCS (Switzerland), IFIN-HH (Romania), CBPF (Brazil), PL-GRID (Poland), and NERSC (USA). We are indebted to the communities behind the multiple open-source software packages on which we depend. Individual groups or members have received support from ARC and ARDC (Australia); AvH Foundation (Germany); EPLANET, Marie Skłodowska-Curie Actions and ERC (European Union); A*MIDEX, ANR, IPhU and Labex P2IO, and Région Auvergne-Rhône-Alpes (France); Key Research Program of Frontier Sciences of CAS, CAS PIFI, CAS CCEPP, Fundamental Research Funds for the Central Universities, and Sci. \& Tech. Program of Guangzhou (China); RFBR, RSF, and Yandex LLC (Russia); GVA, XuntaGal, and GENCAT (Spain); and the Leverhulme Trust, the Royal Society, and UKRI (United Kingdom).

[1] E. Golowich, J. Hewett, S. Pakvasa, and A. A. Petrov, Implications of $D^{0}-\bar{D}^{0}$ mixing for new physics, Phys. Rev. D 76, 095009 (2007).

[2] G. Isidori, Y. Nir, and G. Perez, Flavor physics constraints for physics beyond the standard model, Annu. Rev. Nucl. Part. Sci. 60, 355 (2010).

[3] M. Bobrowski, A. Lenz, J. Riedl, and J. Rohrwild, How large can the SM contribution to CP violation in $D^{0}-\bar{D}^{0}$ mixing be? J. High Energy Phys. 03 (2010) 009.

[4] M. Bona et al. (UTfit Collaboration), Model-independent constraints on $\Delta F=2$ operators and the scale of new physics, J. High Energy Phys. 03 (2008) 049.

[5] P. A. Zyla et al. (Particle Data Group), Review of particle physics, to be published in Prog. Theor. Exp. Phys. 2020, 083C01 (2020).

[6] D. S. Du, Searching for possible large $C P$-violation effects in neutral-charm-meson decays, Phys. Rev. D 34, 3428 (1986).

[7] S. Bergmann, Y. Grossman, Z. Ligeti, Y. Nir, and A. A. Petrov, Lessons from CLEO and FOCUS measurements of $D^{0}-\bar{D}^{0}$ mixing parameters, Phys. Lett. B 486, 418 (2000). 
[8] Y. Amhis et al. (Heavy Flavor Averaging Group), Averages of $b$-hadron, $c$-hadron, and $\tau$-lepton properties as of 2018, updated results and plots available at https://hflav .web.cern.ch.

[9] B. Aubert et al. (BABAR Collaboration), Evidence for $D^{0}-\bar{D}^{0}$ Mixing, Phys. Rev. Lett. 98, 211802 (2007).

[10] M. Staric et al. (Belle Collaboration), Evidence for $D^{0}-\bar{D}^{0}$ Mixing, Phys. Rev. Lett. 98, 211803 (2007).

[11] I. I. Y. Bigi and N. G. Uraltsev, $D^{0}-\bar{D}^{0}$ oscillations as a probe of quark hadron duality, Nucl. Phys. B592, 92 (2001).

[12] A. F. Falk, Y. Grossman, Z. Ligeti, Y. Nir, and A. A. Petrov, The $D^{0}-\bar{D}^{0}$ mass difference from a dispersion relation, Phys. Rev. D 69, 114021 (2004).

[13] A. L. Kagan and L. Silvestrini, Dispersive and absorptive $C P$ violation in $D^{0}-\bar{D}^{0}$ mixing, Phys. Rev. D 103, 053008 (2021).

[14] D. M. Asner et al. (CLEO Collaboration), Search for $D^{0}$ $-\bar{D}^{0}$ mixing in the Dalitz plot analysis of $D^{0} \rightarrow K_{\mathrm{S}}^{0} \pi^{+} \pi^{-}$, Phys. Rev. D 72, 012001 (2005).

[15] T. Peng et al. (Belle Collaboration), Measurement of $D^{0}-\bar{D}^{0}$ mixing and search for indirect $C P$ violation using $D^{0} \rightarrow$ $K_{\mathrm{S}}^{0} \pi^{+} \pi^{-}$decays, Phys. Rev. D 89, 091103 (2014).

[16] P. del Amo Sanchez et al. (BABAR Collaboration), Measurement of $D^{0}-\bar{D}^{0}$ Mixing Parameters using $D^{0} \rightarrow$ $K_{\mathrm{S}}^{0} \pi^{+} \pi^{-}$and $D^{0} \rightarrow K_{\mathrm{S}}^{0} K^{+} K^{-}$Decays, Phys. Rev. Lett. 105, 081803 (2010).

[17] R. Aaij et al. (LHCb collaboration), Model-independent measurement of mixing parameters in $D^{0} \rightarrow K_{\mathrm{S}}^{0} \pi^{+} \pi^{-}$decays, J. High Energy Phys. 04 (2016) 033.

[18] R. Aaij et al. (LHCb Collaboration), Measurement of the Mass Difference Between Neutral Charm-Meson Eigenstates, Phys. Rev. Lett. 122, 231802 (2019).

[19] R. H. Dalitz, On the analysis of $\tau$-meson data and the nature of the $\tau$-meson, Philos. Mag. Ser. 7 44, 1068 (1953).

[20] E. Fabri, A study of $\tau$-meson decay, Nuovo Cimento 11, 479 (1954).
[21] A. Di Canto, J. Garra Ticó, T. Gershon, N. Jurik, M. Martinelli, T. Pilař, S. Stahl, and D. Tonelli, Novel method for measuring charm-mixing parameters using multibody decays, Phys. Rev. D 99, 012007 (2019).

[22] J. Libby et al. (CLEO Collaboration), Model-independent determination of the strong-phase difference between $D^{0}$ and $\bar{D}^{0} \rightarrow K_{\mathrm{S}, \mathrm{L}}^{0} h^{+} h^{-}(h=\pi, K)$ and its impact on the measurement of the CKM angle $\gamma / \phi_{3}$, Phys. Rev. D 82, 112006 (2010).

[23] M. Ablikim et al. (BESIII Collaboration), Model-independent determination of the relative strong-phase difference between $D^{0}$ and $\bar{D}^{0} \rightarrow K_{S, L}^{0} \pi^{+} \pi^{-}$and its impact on the measurement of the CKM angle $\gamma / \phi_{3}$, Phys. Rev. D 101, 112002 (2020).

[24] A. A. Alves Jr. et al. (LHCb Collaboration), The LHCb detector at the LHC, J. Instrum. 3, S08005 (2008).

[25] R. Aaij et al. (LHCb Collaboration), LHCb detector performance, Int. J. Mod. Phys. A 30, 1530022 (2015).

[26] W. D. Hulsbergen, Decay chain fitting with a Kalman filter, Nucl. Instrum. Methods Phys. Res., Sect. A 552, 566 (2005).

[27] N. L. Johnson, Systems of frequency curves generated by methods of translation, Biometrika 36, 149 (1949).

[28] M. Pivk and F. R. Le Diberder, sPlot: A statistical tool to unfold data distributions, Nucl. Instrum. Methods Phys. Res., Sect. A 555, 356 (2005).

[29] I. Adachi et al. (BABAR Collaboration, Belle Collaboration), First Evidence for $\cos 2 \beta>0$ and Resolution of the CKM Unitarity Triangle Ambiguity by a Time-Dependent Dalitz Plot Analysis of $B^{0} \rightarrow D^{(*)} h^{0}$ with $D \rightarrow K_{S}^{0} \pi^{+} \pi^{-}$ Decays, Phys. Rev. Lett. 121, 261801 (2018).

[30] See Supplemental Material at http://link.aps.org/ supplemental/10.1103/PhysRevLett.127.111801 for full fit results and summary of systematic uncertainties.

[31] R. Aaij et al. (LHCb Collaboration), Measurement of the CKM angle $\gamma$ from a combination of $B^{ \pm} \rightarrow D h^{ \pm}$analyses, Phys. Lett. B 726, 151 (2013).

R. Aaij, ${ }^{32}$ C. Abellán Beteta, ${ }^{50}$ T. Ackernley,${ }^{60}$ B. Adeva, ${ }^{46}$ M. Adinolfi, ${ }^{54}$ H. Afsharnia, ${ }^{9}$ C. A. Aidala,${ }^{86}$ S. Aiola, ${ }^{25}$ Z. Ajaltouni, ${ }^{9}$ S. Akar, ${ }^{65}$ J. Albrecht,,${ }^{15}$ F. Alessio, ${ }^{48}$ M. Alexander, ${ }^{59}$ A. Alfonso Albero, ${ }^{45}$ Z. Aliouche, ${ }^{62}$ G. Alkhazov, ${ }^{38}$ P. Alvarez Cartelle, ${ }^{55}$ S. Amato, ${ }^{2}$ Y. Amhis, ${ }^{11}$ L. An, ${ }^{48}$ L. Anderlini, ${ }^{22}$ A. Andreianov, ${ }^{38}$ M. Andreotti, ${ }^{21}$ F. Archilli, ${ }^{17}$ A. Artamonov, ${ }^{44}$ M. Artuso, ${ }^{68} \mathrm{~K}$. Arzymatov ${ }^{42}$ E. Aslanides, ${ }^{10}$ M. Atzeni,${ }^{50}$ B. Audurier, ${ }^{12}$ S. Bachmann, ${ }^{17}$ M. Bachmayer, ${ }^{49}$ J. J. Back, ${ }^{56}$ P. Baladron Rodriguez, ${ }^{46}$ V. Balagura, ${ }^{12}$ W. Baldini ${ }^{21}$ J. Baptista Leite, ${ }^{1}$ R. J. Barlow, ${ }^{62}$ S. Barsuk, ${ }^{11}$ W. Barter, ${ }^{61}$ M. Bartolini, ${ }^{24}$ F. Baryshnikov, ${ }^{83}$ J. M. Basels, ${ }^{14}$ G. Bassi, ${ }^{29}$ B. Batsukh,${ }^{68}$ A. Battig, ${ }^{15}$ A. Bay, ${ }^{49}$ M. Becker ${ }^{15}$ F. Bedeschi, ${ }^{29}$ I. Bediaga, ${ }^{1}$ A. Beiter ${ }^{68}$ V. Belavin, ${ }^{42}$ S. Belin,${ }^{27}$ V. Bellee, ${ }^{49}$ K. Belous,${ }^{44}$ I. Belov, ${ }^{40}$ I. Belyaev, ${ }^{41}$ G. Bencivenni, ${ }^{23}$ E. Ben-Haim, ${ }^{13}$ A. Berezhnoy, ${ }^{40}$ R. Bernet, ${ }^{50}$ D. Berninghoff, ${ }^{17}$ H. C. Bernstein, ${ }^{68}$ C. Bertella ${ }^{48}$ A. Bertolin, ${ }^{28}$ C. Betancourt, ${ }^{50}$ F. Betti, ${ }^{48}$ Ia. Bezshyiko, ${ }^{50}$ S. Bhasin, ${ }^{54}$ J. Bhom,${ }^{35}$ L. Bian,${ }^{73}$ M. S. Bieker, ${ }^{15}$ S. Bifani ${ }^{53}$ P. Billoir ${ }^{13}$ M. Birch ${ }^{61}$ F. C. R. Bishop,${ }^{55}$ A. Bitadze, ${ }^{62}$ A. Bizzeti, ${ }^{22, k}$ M. Bjørn,${ }^{63}$ M. P. Blago, ${ }^{48}$ T. Blake, ${ }^{56}$ F. Blanc, ${ }^{49}$ S. Blusk, ${ }^{68}$ D. Bobulska, ${ }^{59}$ J. A. Boelhauve, ${ }^{15}$ O. Boente Garcia, ${ }^{46}$ T. Boettcher, ${ }^{65}$ A. Boldyrev, ${ }^{82}$ A. Bondar, ${ }^{43}$ N. Bondar, ${ }^{38,48}$ S. Borghi, ${ }^{62}$ M. Borisyak,${ }^{42}$ M. Borsato, ${ }^{17}$ J. T. Borsuk, ${ }^{35}$ S. A. Bouchiba, ${ }^{4, g 9}$ T. J. V. Bowcock, ${ }^{60}$ A. Boyer ${ }^{48}$ C. Bozzi, ${ }^{21}$ M. J. Bradley, ${ }^{61}$ S. Braun ${ }^{66}$ A. Brea Rodriguez,${ }^{46}$ M. Brodski, ${ }^{48}$ J. Brodzicka, ${ }^{35}$ A. Brossa Gonzalo, ${ }^{56}$ D. Brundu, ${ }^{27}$ A. Buonaura, ${ }^{50}$ C. Burr, ${ }^{48}$ A. Bursche, ${ }^{72}$ A. Butkevich, ${ }^{39}$ J. S. Butter, ${ }^{32}$ J. Buytaert, ${ }^{48}$ W. Byczynski, ${ }^{48}$ S. Cadeddu, ${ }^{27}$ H. Cai, ${ }^{73}$ R. Calabrese, ${ }^{21, f}$ L. Calefice, ${ }^{15,13}$ L. Calero Diaz, ${ }^{23}$ S. Cali, ${ }^{23}$ R. Calladine, ${ }^{53}$ M. Calvi, ${ }^{26, j}$ 
M. Calvo Gomez, ${ }^{85}$ P. Camargo Magalhaes ${ }^{54}$ P. Campana, ${ }^{23}$ A. F. Campoverde Quezada, ${ }^{6}$ S. Capelli, ${ }^{26, j}$ L. Capriotti, ${ }^{20, \mathrm{~d}}$ A. Carbone, ${ }^{20, \mathrm{~d}}$ G. Carboni, ${ }^{31}$ R. Cardinale, ${ }^{24}$ A. Cardini, ${ }^{27}$ I. Carli, ${ }^{4}$ P. Carniti, ${ }^{26, j}$ L. Carus, ${ }^{14}$ K. Carvalho Akiba, ${ }^{32}$ A. Casais Vidal, ${ }^{46}$ G. Casse,${ }^{60}$ M. Cattaneo,${ }^{48}$ G. Cavallero, ${ }^{48}$ S. Celani,${ }^{49}$ J. Cerasoli, ${ }^{10}$ A. J. Chadwick, ${ }^{60}$ M. G. Chapman, ${ }^{54}$ M. Charles ${ }^{13} \mathrm{Ph}$. Charpentier, ${ }^{48}$ G. Chatzikonstantinidis, ${ }^{53}$ C. A. Chavez Barajas, ${ }^{60}$ M. Chefdeville, ${ }^{8}$ C. Chen, ${ }^{3}$ S. Chen, ${ }^{4}$ A. Chernov, ${ }^{35}$ V. Chobanova, ${ }^{46}$ S. Cholak, ${ }^{49}$ M. Chrzaszcz, ${ }^{35}$ A. Chubykin, ${ }^{38}$ V. Chulikov, ${ }^{38}$ P. Ciambrone, ${ }^{23}$ M. F. Cicala, ${ }^{56}$ X. Cid Vidal, ${ }^{46}$ G. Ciezarek ${ }^{48}$ P. E. L. Clarke, ${ }^{58}$ M. Clemencic, ${ }^{48}$ H. V. Cliff, ${ }^{55}$ J. Closier, ${ }^{62}$ J. L. Cobbledick, ${ }^{62}$ V. Coco, ${ }^{48}$ J. A. B. Coelho, ${ }^{11}$ J. Cogan, ${ }^{10}$ E. Cogneras, ${ }^{9}$ L. Cojocariu, ${ }^{37}$ P. Collins,${ }^{48}$ T. Colombo, ${ }^{48}$ L. Congedo, ${ }^{19, \mathrm{c}}$ A. Contu, ${ }^{27}$ N. Cooke, ${ }^{53}$ G. Coombs, ${ }^{59}$ G. Corti, ${ }^{48}$ C. M. Costa Sobral,${ }^{56}$ B. Couturier ${ }^{48}$ D. C. Craik, ${ }^{64}$ J. Crkovská, ${ }^{67}$ M. Cruz Torres, ${ }^{1}$ R. Currie, ${ }^{58}$ C. L. Da Silva, ${ }^{67}$ S. Dadabaev ${ }^{83}$ E. Dall'Occo, ${ }^{15}$ J. Dalseno, ${ }^{46}$ C. D'Ambrosio, ${ }^{48}$ A. Danilina, ${ }^{41}$ P. d'Argent ${ }^{48}$ A. Davis, ${ }^{62}$ O. De Aguiar Francisco, ${ }^{62}$ K. De Bruyn, ${ }^{79}$ S. De Capua,${ }^{62}$ M. De Cian, ${ }^{49}$ J. M. De Miranda, ${ }^{1}$ L. De Paula, ${ }^{2}$ M. De Serio, ${ }^{19, c}$ D. De Simone,${ }^{50}$ P. De Simone, ${ }^{23}$ J. A. de Vries,${ }^{80}$ C. T. Dean, ${ }^{67}$ D. Decamp, ${ }^{8}$ L. Del Buono, ${ }^{13}$ B. Delaney,${ }^{55}$ H.-P. Dembinski, ${ }^{15}$ A. Dendek,${ }^{34}$ V. Denysenko, ${ }^{50}$ D. Derkach,${ }^{82}$ O. Deschamps, ${ }^{9}$ F. Desse, ${ }^{11}$ F. Dettori, ${ }^{27, e}$ B. Dey, ${ }^{77}$

A. Di Canto, ${ }^{48}$ A. Di Cicco, ${ }^{23}$ P. Di Nezza, ${ }^{23}$ S. Didenko, ${ }^{83}$ L. Dieste Maronas, ${ }^{46}$ H. Dijkstra, ${ }^{48}$ V. Dobishuk ${ }^{52}$

A. M. Donohoe, ${ }^{18}$ F. Dordei, ${ }^{27}$ A. C. dos Reis, ${ }^{1}$ L. Douglas, ${ }^{59}$ A. Dovbnya, ${ }^{51}$ A. G. Downes, ${ }^{8}$ K. Dreimanis,${ }^{60}$ M. W. Dudek,${ }^{35}$ L. Dufour, ${ }^{48}$ V. Duk,${ }^{78}$ P. Durante, ${ }^{48}$ J. M. Durham,${ }^{67}$ D. Dutta ${ }^{62}$ A. Dziurda ${ }^{35}$ A. Dzyuba ${ }^{38}$ S. Easo, ${ }^{57}$ U. Egede ${ }^{69}$ V. Egorychev, ${ }^{41}$ S. Eidelman, ${ }^{43, v}$ S. Eisenhardt, ${ }^{58}$ S. Ek-In, ${ }^{49}$ L. Eklund, ${ }^{59, w}$ S. Ely, ${ }^{68}$ A. Ene, ${ }^{37}$ E. Epple, ${ }^{67}$ S. Escher, ${ }^{14}$ J. Eschle ${ }^{50}$ S. Esen, ${ }^{13}$ T. Evans, ${ }^{48}$ A. Falabella ${ }^{20}$ J. Fan, ${ }^{3}$ Y. Fan, ${ }^{6}$ B. Fang,${ }^{73}$ S. Farry, ${ }^{60}$ D. Fazzini, ${ }^{26, j}$ M. Féo, ${ }^{48}$ A. Fernandez Prieto, ${ }^{46}$ J. M. Fernandez-tenllado Arribas, ${ }^{45}$ A. D. Fernez, ${ }^{66}$ F. Ferrari, ${ }^{20, d}$ L. Ferreira Lopes, ${ }^{49}$

F. Ferreira Rodrigues, ${ }^{2}$ S. Ferreres Sole,${ }^{32}$ M. Ferrillo,${ }^{50}$ M. Ferro-Luzzi, ${ }^{48}$ S. Filippov, ${ }^{39}$ R. A. Fini, ${ }^{19}$ M. Fiorini, ${ }^{21, f}$ M. Firlej, ${ }^{34}$ K. M. Fischer, ${ }^{63}$ D. S. Fitzgerald, ${ }^{86}$ C. Fitzpatrick, ${ }^{34}$ T. Fiutowski, ${ }^{34}$ A. Fkiaras, ${ }^{48}$ F. Fleuret, ${ }^{12}$ M. Fontana,${ }^{13}$ F. Fontanelli, ${ }^{24, \mathrm{~h}}$ R. Forty, ${ }^{48}$ V. Franco Lima, ${ }^{60}$ M. Franco Sevilla, ${ }^{66}$ M. Frank, ${ }^{48}$ E. Franzoso, ${ }^{21}$ G. Frau, ${ }^{17}$ C. Frei, ${ }^{48}$

D. A. Friday, ${ }^{59}$ J. Fu, ${ }^{25}$ Q. Fuehring, ${ }^{15}$ W. Funk,${ }^{48}$ E. Gabriel, ${ }^{32}$ T. Gaintseva, ${ }^{42}$ A. Gallas Torreira, ${ }^{46}$ D. Galli, ${ }^{20, d}$ S. Gambetta, ${ }^{58,48}$ Y. Gan, ${ }^{3}$ M. Gandelman, ${ }^{2}$ P. Gandini, ${ }^{25}$ Y. Gao, ${ }^{5}$ M. Garau,${ }^{27}$ L. M. Garcia Martin, ${ }^{56}$ P. Garcia Moreno, ${ }^{45}$ J. García Pardiñas, ${ }^{26, j}$ B. Garcia Plana,${ }^{46}$ F. A. Garcia Rosales, ${ }^{12}$ L. Garrido,${ }^{45}$ C. Gaspar, ${ }^{48}$ R. E. Geertsema,${ }^{32}$ D. Gerick,${ }^{17}$ L. L. Gerken, ${ }^{15}$ E. Gersabeck, ${ }^{62}$ M. Gersabeck, ${ }^{62}$ T. Gershon, ${ }^{56}$ D. Gerstel, ${ }^{10}$ Ph. Ghez, ${ }^{8}$ V. Gibson, ${ }^{55}$ H. K. Giemza, ${ }^{36}$ M. Giovannetti, ${ }^{23, p}$ A. Gioventù, ${ }^{46}$ P. Gironella Gironell, ${ }^{45}$ L. Giubega ${ }^{37}$ C. Giugliano, ${ }^{21, f 48}$ K. Gizdov, ${ }^{58}$

E. L. Gkougkousis ${ }^{48}$ V. V. Gligorov, ${ }^{13}$ C. Göbel,${ }^{70}$ E. Golobardes, ${ }^{85}$ D. Golubkov, ${ }^{41}$ A. Golutvin, ${ }^{61,83}$ A. Gomes, ${ }^{1, a}$ S. Gomez Fernandez, ${ }^{45}$ F. Goncalves Abrantes ${ }^{63}$ M. Goncerz, ${ }^{35}$ G. Gong, ${ }^{3}$ P. Gorbounov,${ }^{41}$ I. V. Gorelov, ${ }^{40}$ C. Gotti, ${ }^{26}$ E. Govorkova, ${ }^{48}$ J. P. Grabowski, ${ }^{17}$ T. Grammatico, ${ }^{13}$ L. A. Granado Cardoso, ${ }^{48}$ E. Graugés,${ }^{45}$ E. Graverini,${ }^{49}$ G. Graziani ${ }^{22}$ A. Grecu, ${ }^{37}$ L. M. Greeven, ${ }^{32}$ P. Griffith, ${ }^{21, f}$ L. Grillo, ${ }^{62}$ S. Gromov, ${ }^{83}$ B. R. Gruberg Cazon, ${ }^{63}$ C. Gu, ${ }^{3}$ M. Guarise, ${ }^{21}$ P. A. Günther, ${ }^{17}$ E. Gushchin, ${ }^{39}$ A. Guth, ${ }^{14}$ Y. Guz ${ }^{44}$ T. Gys, ${ }^{48}$ T. Hadavizadeh,${ }^{69}$ G. Haefeli, ${ }^{49}$ C. Haen, ${ }^{48}$ J. Haimberger, ${ }^{48}$ T. Halewood-leagas, ${ }^{60}$ P. M. Hamilton, ${ }^{66}$ J. P. Hammerich,${ }^{60}$ Q. Han, ${ }^{7}$ X. Han,${ }^{17}$ T. H. Hancock,${ }^{63}$

S. Hansmann-Menzemer, ${ }^{17}$ N. Harnew, ${ }^{63}$ T. Harrison, ${ }^{60}$ C. Hasse, ${ }^{48}$ M. Hatch, ${ }^{48}$ J. He, ${ }^{6, b}$ M. Hecker,${ }^{61}$ K. Heijhoff, ${ }^{32}$ K. Heinicke, ${ }^{15}$ A. M. Hennequin, ${ }^{48}$ K. Hennessy, ${ }^{60}$ L. Henry, ${ }^{48}$ J. Heuel, ${ }^{14}$ A. Hicheur, ${ }^{2}$ D. Hill, ${ }^{49}$ M. Hilton,${ }^{62}$ S. E. Hollitt, ${ }^{15}$ J. Hu, ${ }^{17}$ J. Hu, ${ }^{72}$ W. Hu, ${ }^{7}$ X. Hu, ${ }^{3}$ W. Huang, ${ }^{6}$ X. Huang, ${ }^{73}$ W. Hulsbergen, ${ }^{32}$ R. J. Hunter, ${ }^{56}$ M. Hushchyn, ${ }^{82}$ D. Hutchcroft, ${ }^{60}$ D. Hynds, ${ }^{32}$ P. Ibis, ${ }^{15}$ M. Idzik, ${ }^{34}$ D. Ilin, ${ }^{38}$ P. Ilten, ${ }^{65}$ A. Inglessi,${ }^{38}$ A. Ishteev, ${ }^{83}$ K. Ivshin, ${ }^{38}$ R. Jacobsson, ${ }^{48}$ S. Jakobsen,${ }^{48}$ E. Jans,${ }^{32}$ B. K. Jashal, ${ }^{47}$ A. Jawahery, ${ }^{66}$ V. Jevtic,${ }^{15}$ M. Jezabek, ${ }^{35}$ F. Jiang, ${ }^{3}$ M. John, ${ }^{63}$ D. Johnson, ${ }^{48}$ C. R. Jones, ${ }^{55}$ T. P. Jones, ${ }^{56}$ B. Jost, ${ }^{48}$ N. Jurik, ${ }^{48}$ S. Kandybei, ${ }^{51}$ Y. Kang, ${ }^{3}$ M. Karacson, ${ }^{48}$ M. Karpov ${ }^{82}$ F. Keizer, ${ }^{48}$ M. Kenzie, ${ }^{56}$

T. Ketel ${ }^{33}$ B. Khanji, ${ }^{15}$ A. Kharisova, ${ }^{84}$ S. Kholodenko, ${ }^{44}$ T. Kirn, ${ }^{14}$ V. S. Kirsebom, ${ }^{49}$ O. Kitouni, ${ }^{64}$ S. Klaver, ${ }^{32}$ K. Klimaszewski, ${ }^{36}$ S. Koliiev, ${ }^{52}$ A. Kondybayeva, ${ }^{83}$ A. Konoplyannikov, ${ }^{41}$ P. Kopciewicz, ${ }^{34}$ R. Kopecna, ${ }^{17}$ P. Koppenburg, ${ }^{32}$ M. Korolev, ${ }^{40}$ I. Kostiuk, ${ }^{32,52}$ O. Kot, ${ }^{52}$ S. Kotriakhova, ${ }^{21,38}$ P. Kravchenko, ${ }^{38}$ L. Kravchuk, ${ }^{39}$ R. D. Krawczyk, ${ }^{48}$ M. Kreps, ${ }^{56}$ F. Kress, ${ }^{61}$ S. Kretzschmar, ${ }^{14}$ P. Krokovny, ${ }^{43, v}$ W. Krupa,${ }^{34}$ W. Krzemien, ${ }^{36}$ W. Kucewicz, ${ }^{35, t}$ M. Kucharczyk, ${ }^{35}$ V. Kudryavtsev, ${ }^{43, v}$ H. S. Kuindersma, ${ }^{32,33}$ G. J. Kunde, ${ }^{67}$ T. Kvaratskheliya, ${ }^{41}$ D. Lacarrere, ${ }^{48}$ G. Lafferty, ${ }^{62}$ A. Lai, ${ }^{27}$ A. Lampis, ${ }^{27}$ D. Lancierini, ${ }^{50}$ J. J. Lane ${ }^{62}$ R. Lane, ${ }^{54}$ G. Lanfranchi, ${ }^{23}$ C. Langenbruch, ${ }^{14}$ J. Langer, ${ }^{15}$ O. Lantwin, ${ }^{50}$ T. Latham, ${ }^{56}$ F. Lazzari, ${ }^{29, q}$ R. Le Gac, ${ }^{10}$ S. H. Lee, ${ }^{86}$ R. Lefèvre, ${ }^{9}$ A. Leflat, ${ }^{40}$ S. Legotin,${ }^{83}$ O. Leroy, ${ }^{10}$ T. Lesiak, ${ }^{35}$ B. Leverington, ${ }^{17} \mathrm{H} . \mathrm{Li},{ }^{72} \mathrm{~L} . \mathrm{Li},{ }^{63} \mathrm{P} . \mathrm{Li},{ }^{17} \mathrm{~S} . \mathrm{Li},{ }^{7} \mathrm{Y} . \mathrm{Li},{ }^{4} \mathrm{Y} . \mathrm{Li},{ }^{4} \mathrm{Z} . \mathrm{Li},{ }^{68}$ X. Liang, ${ }^{68}$ T. Lin,${ }^{61}$ R. Lindner, ${ }^{48}$ V. Lisovskyi, ${ }^{15}$ R. Litvinov, ${ }^{27}$ G. Liu, ${ }^{72}$ H. Liu, ${ }^{6}$ S. Liu, ${ }^{4}$ A. Loi, ${ }^{27}$ J. Lomba Castro, ${ }^{46}$ I. Longstaff, ${ }^{59}$ J. H. Lopes, ${ }^{2}$ G. H. Lovell, ${ }^{55}$ Y. Lu, ${ }^{4}$ D. Lucchesi, ${ }^{28,1}$ S. Luchuk, ${ }^{39}$ M. Lucio Martinez, ${ }^{32}$ V. Lukashenko, ${ }^{32}$ Y. Luo, ${ }^{3}$ A. Lupato, ${ }^{62}$ E. Luppi ${ }^{21, f}$ O. Lupton, ${ }^{56}$ A. Lusiani,${ }^{29, m}$ X. Lyu, ${ }^{6}$ L. Ma, ${ }^{4}$ R. Ma, ${ }^{6}$ S. Maccolini, ${ }^{20, d}$ F. Machefert, ${ }^{11}$ F. Maciuc, ${ }^{37}$ 
V. Macko, ${ }^{49}$ P. Mackowiak, ${ }^{15}$ S. Maddrell-Mander,${ }^{54}$ O. Madejczyk, ${ }^{34}$ L. R. Madhan Mohan,${ }^{54}$ O. Maev, ${ }^{38}$ A. Maevskiy, ${ }^{82}$ D. Maisuzenko, ${ }^{38}$ M. W. Majewski, ${ }^{34}$ J. J. Malczewski, ${ }^{35}$ S. Malde, ${ }^{63}$ B. Malecki, ${ }^{48}$ A. Malinin, ${ }^{81}$ T. Maltsev, ${ }^{43, v}$ H. Malygina, ${ }^{17}$ G. Manca, ${ }^{27, \mathrm{e}}$ G. Mancinelli, ${ }^{10}$ D. Manuzzi, ${ }^{20, \mathrm{~d}}$ D. Marangotto, ${ }^{25, \mathrm{i}}$ J. Maratas, ${ }^{9, \mathrm{~s}}$ J. F. Marchand, ${ }^{8}$ U. Marconi, ${ }^{20}$ S. Mariani, ${ }^{22, g}$ C. Marin Benito, ${ }^{48}$ M. Marinangeli, ${ }^{49}$ J. Marks, ${ }^{17}$ A. M. Marshall, ${ }^{54}$ P. J. Marshall, ${ }^{60}$ G. Martellotti ${ }^{30}$ L. Martinazzoli, ${ }^{48, j}$ M. Martinelli, ${ }^{26, j}$ D. Martinez Santos ${ }^{46}{ }^{4}$. Martinez Vidal,${ }^{47}$ A. Massafferri, ${ }^{1}$ M. Materok, ${ }^{14}$ R. Matev ${ }^{48}$ A. Mathad ${ }^{50}$ Z. Mathe, ${ }^{48}$ V. Matiunin, ${ }^{41}$ C. Matteuzzi, ${ }^{26}$ K. R. Mattioli, ${ }^{86}$ A. Mauri, ${ }^{32}$ E. Maurice, ${ }^{12}$ J. Mauricio, ${ }^{45}$ M. Mazurek, ${ }^{48}$ M. McCann,${ }^{61}$ L. Mcconnell, ${ }^{18}$ T. H. Mcgrath, ${ }^{62}$ A. McNab,${ }^{62}$ R. McNulty, ${ }^{18}$ J. V. Mead, ${ }^{60}$ B. Meadows, ${ }^{65}$ G. Meier, ${ }^{15}$ N. Meinert, ${ }^{76}$ D. Melnychuk, ${ }^{36}$ S. Meloni, ${ }^{26, j}$ M. Merk, ${ }^{32,80}$ A. Merli, ${ }^{25}$ L. Meyer Garcia, ${ }^{2}$ M. Mikhasenko, ${ }^{48}$ D. A. Milanes, ${ }^{74}$ E. Millard, ${ }^{56}$ M. Milovanovic, ${ }^{48}$ M.-N. Minard, ${ }^{8}$ A. Minotti, ${ }^{21}$ L. Minzoni, ${ }^{21, f}$ S. E. Mitchell, ${ }^{58}$ B. Mitreska, ${ }^{62}$ D. S. Mitzel, ${ }^{48}$ A. Mödden, ${ }^{15}$ R. A. Mohammed, ${ }^{63}$ R. D. Moise,${ }^{61}$ T. Mombächer, ${ }^{46}$ I. A. Monroy, ${ }^{74}$ S. Monteil, ${ }^{9}$ M. Morandin,${ }^{28}$ G. Morello, ${ }^{23}$ M. J. Morello, ${ }^{29, m}$ J. Moron, ${ }^{34}$ A. B. Morris, ${ }^{75}$ A. G. Morris, ${ }^{56}$ R. Mountain, ${ }^{68}$ H. Mu, ${ }^{3}$ F. Muheim, ${ }^{58,48}$ M. Mulder, ${ }^{48}$ D. Müller, ${ }^{48}$ K. Müller, ${ }^{50}$ C. H. Murphy, ${ }^{63}$ D. Murray, ${ }^{62}$ P. Muzzetto, ${ }^{27,48}$ P. Naik, ${ }^{54}$ T. Nakada, ${ }^{49}$ R. Nandakumar, ${ }^{57}$ T. Nanut, ${ }^{49}$ I. Nasteva, ${ }^{2}$ M. Needham, ${ }^{58}$ I. Neri, ${ }^{21}$ N. Neri, ${ }^{25, i}$ S. Neubert, ${ }^{75}$ N. Neufeld,${ }^{48}$ R. Newcombe, ${ }^{61}$ T. D. Nguyen, ${ }^{49}$ C. Nguyen-Mau,${ }^{49, x}$ E. M. Niel,${ }^{11}$ S. Nieswand ${ }^{14}$ N. Nikitin, ${ }^{40}$ N. S. Nolte, ${ }^{64}$ C. Normand,${ }^{8}$ C. Nunez, ${ }^{86}$ A. Oblakowska-Mucha, ${ }^{34}$ V. Obraztsov, ${ }^{44}$ D. P. O'Hanlon, ${ }^{54}$ R. Oldeman, ${ }^{27, e}$ M. E. Olivares, ${ }^{68}$ C. J. G. Onderwater, ${ }^{79}$ R. H. O'neil, ${ }^{58}$ A. Ossowska, ${ }^{35}$ J. M. Otalora Goicochea, ${ }^{2}$ T. Ovsiannikova, ${ }^{41}$ P. Owen, ${ }^{50}$ A. Oyanguren, ${ }^{47}$ B. Pagare, ${ }^{56}$ P. R. Pais ${ }^{48}$ T. Pajero, ${ }^{63}$ A. Palano, ${ }^{19}$ M. Palutan, ${ }^{23}$ Y. Pan ${ }^{62}$ G. Panshin, ${ }^{84}$ A. Papanestis, ${ }^{57}$ M. Pappagallo, ${ }^{19, \mathrm{c}}$ L. L. Pappalardo, ${ }^{21, \mathrm{f}}$ C. Pappenheimer, ${ }^{65} \mathrm{~W}$. Parker, ${ }^{66}$ C. Parkes, ${ }^{62}$ C. J. Parkinson, ${ }^{46}$ B. Passalacqua, ${ }^{21}$ G. Passaleva, ${ }^{22}$ A. Pastore,${ }^{19}$ M. Patel,${ }^{61}$ C. Patrignani, ${ }^{20, d}$ C. J. Pawley,${ }^{80}$ A. Pearce, ${ }^{48}$ A. Pellegrino, ${ }^{32}$ M. Pepe Altarelli, ${ }^{48}$ S. Perazzini, ${ }^{20}$ D. Pereima,${ }^{41}$ P. Perret,${ }^{9}$ I. Petrenko, ${ }^{52}$ M. Petric,${ }^{59,48}$ K. Petridis, ${ }^{54}$ A. Petrolini, ${ }^{24 h}$ A. Petrov, ${ }^{81}$ S. Petrucci, ${ }^{58}$ M. Petruzzo, ${ }^{25}$ T. T. H. Pham, ${ }^{68}$ A. Philippov, ${ }^{42}$ L. Pica, ${ }^{29, m}$ M. Piccini, ${ }^{78}$ B. Pietrzyk,${ }^{8}$ G. Pietrzyk,${ }^{49}$ M. Pili, ${ }^{63}$ D. Pinci, ${ }^{30}$ F. Pisani, ${ }^{48}$ Resmi P. K.,${ }^{10}$ V. Placinta,${ }^{37}$ J. Plews, ${ }^{53}$ M. Plo Casasus, ${ }^{46}$ F. Polci, ${ }^{13}$ M. Poli Lener, ${ }^{23}$ M. Poliakova, ${ }^{68}$ A. Poluektov, ${ }^{10}$ N. Polukhina, ${ }^{83, u}$ I. Polyakov, ${ }^{68}$ E. Polycarpo, ${ }^{2}$ G. J. Pomery, ${ }^{54}$ S. Ponce, ${ }^{48}$ D. Popov ${ }^{6,48}$ S. Popov, ${ }^{42}$ S. Poslavskii, ${ }^{44}$ K. Prasanth,${ }^{35}$ L. Promberger, ${ }^{48}$ C. Prouve, ${ }^{46}$ V. Pugatch, ${ }^{52}$ H. Pullen, ${ }^{63}$ G. Punzi, ${ }^{29, \mathrm{n}}$ H. Qi ${ }^{3}$ W. Qian, ${ }^{6}$ J. Qin, ${ }^{6}$ N. Qin, ${ }^{3}$ R. Quagliani, ${ }^{13}$ B. Quintana, ${ }^{8}$ N. V. Raab, ${ }^{18}$ R. I. Rabadan Trejo, ${ }^{10}$ B. Rachwal, ${ }^{34}$ J. H. Rademacker, ${ }^{54}$ M. Rama, ${ }^{29}$ M. Ramos Pernas, ${ }^{56}$ M. S. Rangel, ${ }^{2}$ F. Ratnikov, ${ }^{42,82}$ G. Raven, ${ }^{33}$ M. Reboud ${ }^{8}$ F. Redi,${ }^{49}$ F. Reiss, ${ }^{62}$ C. Remon Alepuz,${ }^{47}$ Z. Ren, ${ }^{3}$ V. Renaudin,${ }^{63}$ R. Ribatti, ${ }^{29}$ S. Ricciardi,${ }^{57}$ K. Rinnert, ${ }^{60}$ P. Robbe, ${ }^{11}$ G. Robertson, ${ }^{58}$ A. B. Rodrigues,${ }^{49}$ E. Rodrigues, ${ }^{60}$ J. A. Rodriguez Lopez, ${ }^{74}$ A. Rollings, ${ }^{63}$ P. Roloff, ${ }^{48}$ V. Romanovskiy, ${ }^{44}$ M. Romero Lamas, ${ }^{46}$ A. Romero Vidal, ${ }^{46}$ J. D. Roth, ${ }^{86}$ M. Rotondo, ${ }^{23}$ M. S. Rudolph, ${ }^{68}$ T. Ruf, ${ }^{48}$ J. Ruiz Vidal, ${ }^{47}$ A. Ryzhikov, ${ }^{82}$ J. Ryzka, ${ }^{34}$ J. J. Saborido Silva, ${ }^{46}$ N. Sagidova,${ }^{38}$ N. Sahoo, ${ }^{56}$ B. Saitta, ${ }^{27, e}$ M. Salomoni, ${ }^{48}$ D. Sanchez Gonzalo, ${ }^{45}$ C. Sanchez Gras, ${ }^{32}$ R. Santacesaria,${ }^{30}$ C. Santamarina Rios, ${ }^{46}$ M. Santimaria, ${ }^{23}$ E. Santovetti, ${ }^{31, p}$ D. Saranin, ${ }^{83}$ G. Sarpis, ${ }^{59}$ M. Sarpis, ${ }^{75}$ A. Sarti, ${ }^{30}$ C. Satriano,${ }^{30,0}$ A. Satta, ${ }^{31}$ M. Saur, ${ }^{15}$ D. Savrina, ${ }^{41,40}$ H. Sazak, ${ }^{9}$ L. G. Scantlebury Smead, ${ }^{63}$ A. Scarabotto, ${ }^{13}$ S. Schael, ${ }^{14}$ M. Schiller, ${ }^{59}$ H. Schindler ${ }^{48}$ M. Schmelling, ${ }^{16}$ B. Schmidt, ${ }^{48}$ O. Schneider, ${ }^{49}$ A. Schopper, ${ }^{48}$ M. Schubiger, ${ }^{32}$ S. Schulte, ${ }^{49}$ M. H. Schune, ${ }^{11}$ R. Schwemmer, ${ }^{48}$ B. Sciascia, ${ }^{23}$ S. Sellam, ${ }^{46}$ A. Semennikov, ${ }^{41}$ M. Senghi Soares, ${ }^{33}$ A. Sergi, ${ }^{24}$ N. Serra,${ }^{50}$ L. Sestini, ${ }^{28}$ A. Seuthe, ${ }^{15}$ P. Seyfert, ${ }^{48}$ Y. Shang, ${ }^{5}$

D. M. Shangase, ${ }^{86}$ M. Shapkin, ${ }^{44}$ I. Shchemerov, ${ }^{83}$ L. Shchutska, ${ }^{49}$ T. Shears, ${ }^{60}$ L. Shekhtman, ${ }^{43, v}$ Z. Shen, ${ }^{5}$

V. Shevchenko, ${ }^{81}$ E. B. Shields, ${ }^{26, j}$ E. Shmanin, ${ }^{83}$ J. D. Shupperd, ${ }^{68}$ B. G. Siddi, ${ }^{21}$ R. Silva Coutinho, ${ }^{50}$ G. Simi, ${ }^{28}$

S. Simone, ${ }^{19, \mathrm{c}}$ N. Skidmore, ${ }^{62}$ T. Skwarnicki, ${ }^{68}$ M. W. Slater, ${ }^{53}$ I. Slazyk, ${ }^{21, \mathrm{f}}$ J. C. Smallwood, ${ }^{63}$ J. G. Smeaton, ${ }^{55}$ A. Smetkina, ${ }^{41}$ E. Smith, ${ }^{50}$ M. Smith, ${ }^{61}$ A. Snoch, ${ }^{32}$ M. Soares, ${ }^{20}$ L. Soares Lavra, ${ }^{9}$ M. D. Sokoloff, ${ }^{65}$ F. J. P. Soler, ${ }^{59}$ A. Solovev, ${ }^{38}$ I. Solovyev, ${ }^{38}$ F. L. Souza De Almeida,${ }^{2}$ B. Souza De Paula, ${ }^{2}$ B. Spaan, ${ }^{15}$ E. Spadaro Norella, ${ }^{25, i}$ P. Spradlin, ${ }^{59}$ F. Stagni, ${ }^{48}$ M. Stahl, ${ }^{65}$ S. Stahl, ${ }^{48}$ P. Stefko, ${ }^{49}$ O. Steinkamp, ${ }^{50,83}$ O. Stenyakin, ${ }^{44}$ H. Stevens, ${ }^{15}$ S. Stone, ${ }^{68}$ M. E. Stramaglia, ${ }^{49}$ M. Straticiuc, ${ }^{37}$ D. Strekalina, ${ }^{83}$ F. Suljik, ${ }^{63}$ J. Sun, ${ }^{27}$ L. Sun,${ }^{73}$ Y. Sun, ${ }^{66}$ P. Svihra, ${ }^{62}$ P. N. Swallow, ${ }^{53}$ K. Swientek, ${ }^{34}$ A. Szabelski, ${ }^{36}$ T. Szumlak, ${ }^{34}$ M. Szymanski, ${ }^{48}$ S. Taneja, ${ }^{62}$ A. R. Tanner, ${ }^{54}$ A. Terentev, ${ }^{83}$ F. Teubert, ${ }^{48}$ E. Thomas, ${ }^{48}$ K. A. Thomson, ${ }^{60}$ V. Tisserand, ${ }^{9}$ S. T' Jampens, ${ }^{8}$ M. Tobin, ${ }^{4}$ L. Tomassetti, ${ }^{21, f}$ D. Torres Machado, ${ }^{1}$ D. Y. Tou, ${ }^{13}$ M. T. Tran, ${ }^{49}$ E. Trifonova,${ }^{83}$ C. Trippl,,${ }^{49}$ G. Tuci,${ }^{29, n}$ A. Tully, ${ }^{49}$ N. Tuning, ${ }^{32,48}$ A. Ukleja, ${ }^{36}$ D. J. Unverzagt,${ }^{17}$ E. Ursov, ${ }^{83}$ A. Usachov, ${ }^{32}$ A. Ustyuzhanin, ${ }^{42,82}$ U. Uwer, ${ }^{17}$ A. Vagner,${ }^{84}$ V. Vagnoni, ${ }^{20}$ A. Valassi, ${ }^{48}$ G. Valenti, ${ }^{20}$ N. Valls Canudas, ${ }^{85}$ M. van Beuzekom, ${ }^{32}$ M. Van Dijk, ${ }^{49}$ E. van Herwijnen, ${ }^{83}$ C. B. Van Hulse, ${ }^{18}$ M. van Veghel, ${ }^{79}$ R. Vazquez Gomez, ${ }^{46}$ P. Vazquez Regueiro, ${ }^{46}$ C. Vázquez Sierra ${ }^{48}$ S. Vecchi, ${ }^{21}$ J. J. Velthuis, ${ }^{54}$ M. Veltri, ${ }^{22, r}$ A. Venkateswaran, ${ }^{68}$ M. Veronesi, ${ }^{32}$ M. Vesterinen, ${ }^{56}$ D. Vieira, ${ }^{65}$ M. Vieites Diaz, ${ }^{49}$ H. Viemann, ${ }^{76}$ X. Vilasis-Cardona,${ }^{85}$ 
E. Vilella Figueras, ${ }^{60}$ A. Villa, ${ }^{20}$ P. Vincent, ${ }^{13}$ D. Vom Bruch,,${ }^{10}$ A. Vorobyev, ${ }^{38}$ V. Vorobyev, ${ }^{43, v}$ N. Voropaev, ${ }^{38}$ K. Vos,${ }^{80}$ R. Waldi, ${ }^{17}$ J. Walsh, ${ }^{29}$ C. Wang, ${ }^{17}$ J. Wang,,${ }^{5}$ J. Wang, ${ }^{4}$ J. Wang, ${ }^{3}$ J. Wang, ${ }^{73}$ M. Wang, ${ }^{3}$ R. Wang, ${ }^{54}$ Y. Wang, ${ }^{7}$ Z. Wang, ${ }^{50}$ Z. Wang, ${ }^{3}$ H. M. Wark, ${ }^{60}$ N. K. Watson, ${ }^{53}$ S. G. Weber, ${ }^{13}$ D. Websdale, ${ }^{61}$ C. Weisser, ${ }^{64}$ B. D. C. Westhenry,${ }^{54}$ D. J. White, ${ }^{62}$ M. Whitehead ${ }^{54}$ D. Wiedner, ${ }^{15}$ G. Wilkinson,${ }^{63}$ M. Wilkinson, ${ }^{68}$ I. Williams, ${ }^{55}$ M. Williams,${ }^{64}$ M. R. J. Williams,${ }^{58}$ F. F. Wilson, ${ }^{57}$ W. Wislicki, ${ }^{36}$ M. Witek, ${ }^{35}$ L. Witola, ${ }^{17}$ G. Wormser, ${ }^{11}$ S. A. Wotton, ${ }^{55} \mathrm{H}$. Wu, ${ }^{68}$ K. Wyllie, ${ }^{48}$ Z. Xiang, ${ }^{6}$ D. Xiao, ${ }^{7}$ Y. Xie, ${ }^{7}$ A. Xu, ${ }^{5}$ J. Xu, ${ }^{6}$ L. Xu, ${ }^{3}$ M. Xu, ${ }^{7}$ Q. Xu, ${ }^{6}$ Z. Xu, ${ }^{5}$ Z. Xu, ${ }^{6}$ D. Yang, ${ }^{3}$ S. Yang, ${ }^{6}$ Y. Yang, ${ }^{6}$ Z. Yang, ${ }^{3}$ Z. Yang, ${ }^{66}$ Y. Yao, ${ }^{68}$ L. E. Yeomans, ${ }^{60}$ H. Yin, ${ }^{7}$ J. Yu, ${ }^{71}$ X. Yuan, ${ }^{68}$ O. Yushchenko, ${ }^{44}$ E. Zaffaroni, ${ }^{49}$ M. Zavertyaev, ${ }^{16, u}$ M. Zdybal, ${ }^{35}$ O. Zenaiev, ${ }^{48}$ M. Zeng, ${ }^{3}$ D. Zhang, ${ }^{7}$ L. Zhang, ${ }^{3}$ S. Zhang, ${ }^{5}$ Y. Zhang, ${ }^{5}$ Y. Zhang, ${ }^{63}$ A. Zharkova, ${ }^{83}$

A. Zhelezov, ${ }^{17}$ Y. Zheng, ${ }^{6}$ X. Zhou, ${ }^{6}$ Y. Zhou, ${ }^{6}$ X. Zhu, ${ }^{3}$ Z. Zhu, ${ }^{6}$ V. Zhukov, ${ }^{14,40}$ J. B. Zonneveld, ${ }^{58}$ Q. Zou, ${ }^{4}$ S. Zucchelli, ${ }^{20, d}$ D. Zuliani, ${ }^{28}$ and G. Zunica ${ }^{62}$

(LHCb Collaboration)

${ }^{1}$ Centro Brasileiro de Pesquisas Físicas (CBPF), Rio de Janeiro, Brazil

${ }^{2}$ Universidade Federal do Rio de Janeiro (UFRJ), Rio de Janeiro, Brazil

${ }^{3}$ Center for High Energy Physics, Tsinghua University, Beijing, China

${ }^{4}$ Institute Of High Energy Physics (IHEP), Beijing, China

${ }^{5}$ School of Physics State Key Laboratory of Nuclear Physics and Technology, Peking University, Beijing, China

${ }^{6}$ University of Chinese Academy of Sciences, Beijing, China

${ }^{7}$ Institute of Particle Physics, Central China Normal University, Wuhan, Hubei, China

${ }^{8}$ Univ. Savoie Mont Blanc, CNRS, IN2P3-LAPP, Annecy, France

${ }^{9}$ Université Clermont Auvergne, CNRS/IN2P3, LPC, Clermont-Ferrand, France

${ }^{10}$ Aix Marseille Univ, CNRS/IN2P3, CPPM, Marseille, France

${ }^{11}$ Université Paris-Saclay, CNRS/IN2P3, IJCLab, Orsay, France

${ }^{12}$ Laboratoire Leprince-Ringuet, CNRS/IN2P3, Ecole Polytechnique, Institut Polytechnique de Paris, Palaiseau, France

${ }^{13}$ LPNHE, Sorbonne Université, Paris Diderot Sorbonne Paris Cité, CNRS/IN2P3, Paris, France

${ }^{14}$ I. Physikalisches Institut, RWTH Aachen University, Aachen, Germany

${ }^{15}$ Fakultät Physik, Technische Universität Dortmund, Dortmund, Germany

${ }^{16}$ Max-Planck-Institut für Kernphysik (MPIK), Heidelberg, Germany

${ }^{17}$ Physikalisches Institut, Ruprecht-Karls-Universität Heidelberg, Heidelberg, Germany

${ }^{18}$ School of Physics, University College Dublin, Dublin, Ireland

${ }^{19}$ INFN Sezione di Bari, Bari, Italy

${ }^{20}$ INFN Sezione di Bologna, Bologna, Italy

${ }^{21}$ INFN Sezione di Ferrara, Ferrara, Italy

${ }^{22}$ INFN Sezione di Firenze, Firenze, Italy

${ }^{23}$ INFN Laboratori Nazionali di Frascati, Frascati, Italy

${ }^{24}$ INFN Sezione di Genova, Genova, Italy

${ }^{25}$ INFN Sezione di Milano, Milano, Italy

${ }^{26}$ INFN Sezione di Milano-Bicocca, Milano, Italy

${ }^{27}$ INFN Sezione di Cagliari, Monserrato, Italy

${ }^{28}$ Universita degli Studi di Padova, Universita e INFN, Padova, Padova, Italy

${ }^{29}$ INFN Sezione di Pisa, Pisa, Italy

${ }^{30}$ INFN Sezione di Roma La Sapienza, Roma, Italy

${ }^{31}$ INFN Sezione di Roma Tor Vergata, Roma, Italy

${ }^{32}$ Nikhef National Institute for Subatomic Physics, Amsterdam, Netherlands

${ }^{33}$ Nikhef National Institute for Subatomic Physics and VU University Amsterdam, Amsterdam, Netherlands

${ }^{34}$ AGH-University of Science and Technology, Faculty of Physics and Applied Computer Science, Kraków, Poland

${ }^{35}$ Henryk Niewodniczanski Institute of Nuclear Physics Polish Academy of Sciences, Kraków, Poland

${ }^{36}$ National Center for Nuclear Research (NCBJ), Warsaw, Poland

${ }^{37}$ Horia Hulubei National Institute of Physics and Nuclear Engineering, Bucharest-Magurele, Romania

${ }^{38}$ Petersburg Nuclear Physics Institute NRC Kurchatov Institute (PNPI NRC KI), Gatchina, Russia

${ }^{39}$ Institute for Nuclear Research of the Russian Academy of Sciences (INR RAS), Moscow, Russia

${ }^{40}$ Institute of Nuclear Physics, Moscow State University (SINP MSU), Moscow, Russia

${ }^{41}$ Institute of Theoretical and Experimental Physics NRC Kurchatov Institute (ITEP NRC KI), Moscow, Russia

${ }^{42}$ Yandex School of Data Analysis, Moscow, Russia

${ }^{43}$ Budker Institute of Nuclear Physics (SB RAS), Novosibirsk, Russia 
${ }^{44}$ Institute for High Energy Physics NRC Kurchatov Institute (IHEP NRC KI), Protvino, Russia, Protvino, Russia

${ }^{45}$ ICCUB, Universitat de Barcelona, Barcelona, Spain

${ }^{46}$ Instituto Galego de Física de Altas Enerxías (IGFAE), Universidade de Santiago de Compostela, Santiago de Compostela, Spain

${ }^{47}$ Instituto de Fisica Corpuscular, Centro Mixto Universidad de Valencia-CSIC, Valencia, Spain

${ }^{48}$ European Organization for Nuclear Research (CERN), Geneva, Switzerland

${ }^{49}$ Institute of Physics, Ecole Polytechnique Fédérale de Lausanne (EPFL), Lausanne, Switzerland

${ }^{50}$ Physik-Institut, Universität Zürich, Zürich, Switzerland

${ }^{51}$ NSC Kharkiv Institute of Physics and Technology (NSC KIPT), Kharkiv, Ukraine

${ }^{52}$ Institute for Nuclear Research of the National Academy of Sciences (KINR), Kyiv, Ukraine

${ }^{53}$ University of Birmingham, Birmingham, United Kingdom

${ }^{54}$ H.H. Wills Physics Laboratory, University of Bristol, Bristol, United Kingdom

${ }^{55}$ Cavendish Laboratory, University of Cambridge, Cambridge, United Kingdom

${ }^{56}$ Department of Physics, University of Warwick, Coventry, United Kingdom

${ }^{57}$ STFC Rutherford Appleton Laboratory, Didcot, United Kingdom

${ }^{58}$ School of Physics and Astronomy, University of Edinburgh, Edinburgh, United Kingdom

${ }^{59}$ School of Physics and Astronomy, University of Glasgow, Glasgow, United Kingdom

${ }^{60}$ Oliver Lodge Laboratory, University of Liverpool, Liverpool, United Kingdom

${ }^{61}$ Imperial College London, London, United Kingdom

${ }^{62}$ Department of Physics and Astronomy, University of Manchester, Manchester, United Kingdom

${ }^{63}$ Department of Physics, University of Oxford, Oxford, United Kingdom

${ }^{64}$ Massachusetts Institute of Technology, Cambridge, Massachusetts, USA

${ }^{65}$ University of Cincinnati, Cincinnati, Ohio, USA

${ }^{66}$ University of Maryland, College Park, Maryland, USA

${ }^{67}$ Los Alamos National Laboratory (LANL), Los Alamos, New Mexico, USA

${ }^{68}$ Syracuse University, Syracuse, New York, USA

${ }^{69}$ School of Physics and Astronomy, Monash University, Melbourne, Australia

(associated with Department of Physics, University of Warwick, Coventry, United Kingdom)

${ }^{70}$ Pontifícia Universidade Católica do Rio de Janeiro (PUC-Rio), Rio de Janeiro, Brazil

[associated with Universidade Federal do Rio de Janeiro (UFRJ), Rio de Janeiro, Brazil]

${ }^{71}$ Physics and Micro Electronic College, Hunan University, Changsha City, China

(associated with Institute of Particle Physics, Central China Normal University, Wuhan, Hubei, China)

${ }^{72}$ Guangdong Provincial Key Laboratory of Nuclear Science, Guangdong-Hong Kong Joint Laboratory of Quantum Matter,

Institute of Quantum Matter, South China Normal University, Guangzhou, China

(associated with Center for High Energy Physics, Tsinghua University, Beijing, China)

${ }^{73}$ School of Physics and Technology, Wuhan University, Wuhan, China

(associated with Center for High Energy Physics, Tsinghua University, Beijing, China)

${ }^{74}$ Departamento de Fisica, Universidad Nacional de Colombia, Bogota, Colombia

(associated with LPNHE, Sorbonne Université, Paris Diderot Sorbonne Paris Cité, CNRS/IN2P3, Paris, France)

${ }^{75}$ Universität Bonn-Helmholtz-Institut für Strahlen und Kernphysik, Bonn, Germany

(associated with Physikalisches Institut, Ruprecht-Karls-Universität Heidelberg, Heidelberg, Germany)

${ }^{76}$ Institut für Physik, Universität Rostock, Rostock, Germany

(associated with Physikalisches Institut, Ruprecht-Karls-Universität Heidelberg, Heidelberg, Germany)

${ }^{77}$ Eotvos Lorand University, Budapest, Hungary

[associated with European Organization for Nuclear Research (CERN), Geneva, Switzerland]

${ }^{78}$ INFN Sezione di Perugia, Perugia, Italy

(associated with INFN Sezione di Ferrara, Ferrara, Italy)

${ }^{79}$ Van Swinderen Institute, University of Groningen, Groningen, Netherlands

(associated with Nikhef National Institute for Subatomic Physics, Amsterdam, Netherlands)

${ }^{80}$ Universiteit Maastricht, Maastricht, Netherlands

(associated with Nikhef National Institute for Subatomic Physics, Amsterdam, Netherlands)

${ }^{81}$ National Research Centre Kurchatov Institute, Moscow, Russia

[associated with Institute of Theoretical and Experimental Physics NRC Kurchatov Institute (ITEP NRC KI), Moscow, Russia]

${ }^{82}$ National Research University Higher School of Economics, Moscow, Russia

(associated with Yandex School of Data Analysis, Moscow, Russia)

${ }^{83}$ National University of Science and Technology "MISIS", Moscow, Russia

[associated with Institute of Theoretical and Experimental Physics NRC Kurchatov Institute (ITEP NRC KI), Moscow, Russia]

${ }^{84}$ National Research Tomsk Polytechnic University, Tomsk, Russia

[associated with Institute of Theoretical and Experimental Physics NRC Kurchatov Institute (ITEP NRC KI), Moscow, Russia] 


\footnotetext{
${ }^{85}$ DS4DS, La Salle, Universitat Ramon Llull, Barcelona, Spain (associated with ICCUB, Universitat de Barcelona, Barcelona, Spain)

${ }^{86}$ University of Michigan, Ann Arbor, MI, USA

(associated with Syracuse University, Syracuse, New York, USA)
}

aAlso at Universidade Federal do Triângulo Mineiro (UFTM), Uberaba-MG, Brazil.

${ }^{\mathrm{b}}$ Also at Hangzhou Institute for Advanced Study, UCAS, Hangzhou, China.

${ }^{\mathrm{c}}$ Also at Università di Bari, Bari, Italy.

${ }^{\mathrm{d}}$ Also at Università di Bologna, Bologna, Italy.

${ }^{\mathrm{e}}$ Also at Università di Cagliari, Cagliari, Italy.

${ }^{\mathrm{f}}$ Also at Università di Ferrara, Ferrara, Italy.

${ }^{\mathrm{g}}$ Also at Università di Firenze, Firenze, Italy.

${ }^{\mathrm{h}}$ Also at Università di Genova, Genova, Italy.

${ }^{i}$ Also at Università degli Studi di Milano, Milano, Italy.

${ }^{\mathrm{j}}$ Also at Università di Milano Bicocca, Milano, Italy.

${ }^{\mathrm{k}}$ Also at Università di Modena e Reggio Emilia, Modena, Italy.

${ }^{1}$ Also at Università di Padova, Padova, Italy.

${ }^{\mathrm{m}}$ Also at Scuola Normale Superiore, Pisa, Italy.

${ }^{\mathrm{n}}$ Also at Università di Pisa, Pisa, Italy.

${ }^{\circ}$ Also at Università della Basilicata, Potenza, Italy.

${ }^{\mathrm{p}}$ Also at Università di Roma Tor Vergata, Roma, Italy.

${ }^{\mathrm{q}}$ Also at Università di Siena, Siena, Italy.

${ }^{\mathrm{r}}$ Also at Università di Urbino, Urbino, Italy.

${ }^{\mathrm{s}}$ Also at MSU-Iligan Institute of Technology (MSU-IIT), Iligan, Philippines.

${ }^{t}$ Also at AGH-University of Science and Technology, Faculty of Computer Science, Electronics and Telecommunications, Kraków, Poland.

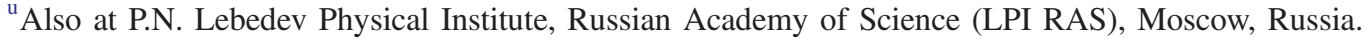

${ }^{v}$ Also at Novosibirsk State University, Novosibirsk, Russia.

${ }^{\mathrm{w}}$ Also at Department of Physics and Astronomy, Uppsala University, Uppsala, Sweden.

${ }^{\mathrm{x}}$ Also at Hanoi University of Science, Hanoi, Vietnam. 